\section{Roche breaks with PacBio, opts for Genia}

Roche will continue to pursue the clinical sequencing market despite scrapping a three-year-old development deal with Pacific Biosciences of Menlo Park, California in December. Neil Gunn, head of Roche's Sequencing Solutions unit, said in an announcement that the company will "actively pursue multiple technologies and commercial strategies" to achieve its long-held goal of market leadership in clinical diagnostics. Pacific Biosciences' single-molecule, realtime technology will no longer be part of the equation, however. Instead, the Basel-based pharma will focus development efforts on the nanopore technology it gained through its $\$ 350$ million acquisition of Santa Clara, California-based Genia Technologies in 2014. Roche is also preparing to launch cancer screening assays to detect circulating tumor DNA. Roche obtained the assays when it bought CAPP Medical, a privately held genomics company founded by Stanford University oncologists last year. In the deal with Pacific Biosciences, Roche had envisioned deploying assays on the biotech's Sequel sequencing instrument. With the deal wound up, Roche is now free to focus its energies elsewhere, while Pacific Biosciences is no longer held to its exclusive relationship with Roche and can court other partners. Pacific Biosciences CEO Michael Hunkapiller said in a statement that he was "disappointed" with Roche's decision, but that the company is "prepared to immediately pursue opportunities" in the clinical research and sequencing market, with a focus on clients that offer laboratory-developed tests. Roche has long coveted a leadership role in the clinical sequencing market and has been particularly acquisitive in the past two years, snatching up Signature Diagnostics, a German translational oncology and genomics company, and Bina Technologies, a genomics analysis provider in Belmont, California, in addition to CAPP Medical and Genia. Roche also acquired Ariosa Diagnostics, a provider of sequencing-based noninvasive prenatal testing located in San Jose, California, in 2014. So far, Roche's attempts to corner the market have been frustrated, notably when it attempted a hostile takeover of San Diego, Californiabased Illumina in 2012 for over \$6 billion, an effort that ended with Illumina shareholders voting against Roche's slate of proposed new candidates to the company's board of directors. In 2013, Roche also wound down its 454 Life Sciences business unit, six years after acquiring the Branford, Connecticut-based company for $\$ 155$ million, citing the noncompetitiveness of its technology in a market dominated by Illumina, Thermo Fisher Scientific and other sequencing players. 454 was widely seen as the Swiss pharma's first foray into the nextgeneration sequencing space. at the base of the spinal cord, whereas most antisense drugs are given systemically. That route of administration allows for lower doses and fewer side effects. But it means Spinraza is not the best test of Ionis' technology more broadly. "This was the lowest-hanging fruit, so to speak," says Stein.

According to Paul Matteis, a biotech analyst at Leerink Partners in New York, the technology's application will face a bigger test with two other late-stage Ionis drug candidates: IONIS$\mathrm{TTR}_{\mathrm{Rx}}$, which reduces levels of the transport protein transthyretin, and volanesorsen, which blocks production of apolipoprotein C-III. Both are currently in phase 3 trials for ultrarare hereditary conditions. Results are expected later this year.

Adverse safety signals almost derailed both therapies. Last year, Ionis and its partner, GlaxoSmithKline, scrapped plans to test IONIS-TTR $_{\mathrm{Rx}}$ in people with aggregated transthyretin protein in the heart, a condition known as familial amyloid cardiomyopathy, after some patients experienced extremely low blood platelet levels while on the drug. The same problem was also seen in trials of volanesorsen. Ionis went ahead with its trials of IONIS-TTR $\mathrm{Rx}$ in patients with familial amyloid polyneuropathy (FAP), a related condition that results from transthyretin buildup in the nerves, and volanesorsen in patients with familial chylomicronemia syndrome (FCS), a disease caused by dysfunctional triglyceride clearance. But the company added regular platelet testing so that patients whose counts started to drop could go off the medicine.

Importantly, the thrombocytopenia events do not appear to be a platform-wide issue with the technology, says Wade Walke, head of communications and investor relations at Ionis. According to Walke, the company has a paper coming out involving more than 1,500 people who have received any of 16 different antisense drugs. Serious platelet declines weren't seen in a single patient. What's more, fluctuations in platelet counts are now understood to be somewhat common in patients with FCS, and there seemed to be a unique problem of anti-platelet antibodies in the patients with FAP.

Nonetheless, the FDA was concerned enough about the link to thrombocytopenia to require a label warning of potential bleeding complications. Spinraza's approval calls for platelet levels to be measured prior to each administration of the drug. A full course involves three doses two weeks apart, followed by a fourth dose a month later and maintenance dosing every four months thereafter.

All those doses won't come cheap. At about $\$ 125,000$ per intrathecal injection, Spinraza will cost $\$ 750,000$ in its first year and $\$ 375,000$ in every year that follows. As with most therapies for rare diseases, insurance companies are expected to cover the price, especially for the more severe and earlier-onset forms of the disease that afflict babies before their first birthday. However, for the $10 \%$ of SMA patients who don't develop muscle weakness until later in childhood or even as adults, Matteis thinks reimbursement might be more challenging. That's why, when he saw the high sticker price of Spinraza, he adjusted his peak sales forecast downward from $\$ 3$ billion to $\$ 2.6$ billionalthough the large first-year bill should yield an "excellent launch," he says.

Further validation for antisense approaches beyond SMA came two weeks after Spinraza’s approval. On January 6, Basel-based Novartis entered a co-development pact with Ionis worth up to $\$ 1.6$ billion if all milestones are met. Ionis and its wholly owned subsidiary Akcea Therapeutics, of Cambridge, Massachusetts, will receive $\$ 225$ million in near-term payments in exchange for commercialization rights to two experimental antisense drugs for treating lipid disorders. One is designed to reduce lipoprotein (a), a cholesterol component linked to heart attacks and stroke; the other, like volanesorsen, targets the apolipoprotein C-III involved in regulating triglyceride levels, which are implicated in type 2 diabetes and other metabolic abnormalities. Each is expected to start phase 2, dose-ranging studies in the first half of this year.

Both therapies incorporate Ionis' nextgeneration LICA technology. Short for ligandconjugated antisense, LICA involves attaching $\mathrm{N}$-acetyl-D-galactosamine to the therapeutic oligonucleotide. This sugar molecule enhances liver uptake, which increases the potency of the antisense molecule and allows lower doses or less frequent administration. "Big pharma is paying extra for this chemistry improvement," says Dirk Haussecker, an oligonucleotide therapeutics analyst and consultant in Rastatt, Germany.

Ionis will need as much money as it can get as it tries to bring another three dozen or so clinical and preclinical drug candidates to market. That may continue to be a bumpy ride. Just last month, company executives and their partners at Biogen announced that they were discontinuing development of an antisense drug for treating a form of myotonic dystrophy after the early-stage therapy didn't prove potent enough in phase $1 / 2$ testing to merit further investment.

Haussecker describes the Novartis partnership as a "stamp of approval" for Ionis, and Stein agrees. "People are getting the sense that these drugs really can do something," he says. "It's not just a lot of talk-and I think they're right."

Elie Dolgin Somerville, Massachusetts 